\title{
Programa de técnicas metacognitivas y la mejora de los niveles de comprensión lectora de los estudiantes de educación técnica
}

\author{
Program of metacognitive techniques and the improvement of levels of \\ reading comprehension of the students of technical education
}

\author{
Aldo Aguayo Meléndez ${ }^{1, *}$ \\ ${ }^{1}$ IESTP Franklin Roosevelt, Lima.
}

\section{RESUMEN}

El presente estudio está orientado a determinar la influencia del programa IMA en la comprensión lectora de los estudiantes de Educación Técnica. Se utilizó una investigación tipo aplicativo, cuasi experimental y utilizando el diseño de cuatro grupos de Solomon. Se llevó a cabo un conjunto de actividades, relacionados a los niveles de comprensión lectora y técnicas metacognitivas, ya que en cada una de las 12 sesiones del programa IMA se aplicó pruebas de lectura y técnicas metacognitivas. Por medio de un muestreo no probabilístico se eligió una muestra de dos grupos experimentales, cada uno con 27 estudiantes, y dos grupos de control, con 24 y 31 estudiantes, respectivamente. La discusión de los resultados determinaron que los estudiantes, participantes en dicho programa, evolucionaron sus habilidades lectoras, basados en la comparación de los resultados del pre y pos test, fortaleciendo la idea de que las habilidades metacognitivas y la comprensión lectora tienen sentido, si se piensan en función del aprendizaje. Los estudiantes incrementaron de manera significativa sus habilidades metacognitivas, ya que en el primer grupo experimental, a partir de los puntos comparativos, hubo un incremento de 4,45 puntos y en el segundo 4,15 puntos. Por tanto, la propuesta permite al estudiante reflexionar sobre su compromiso académico, la capacidad de comprensión y autorregulación que le permitirá mejorar su formación técnico profesional.

Palabras clave: Programa, comprensión lectora, habilidades metacognitivas, técnicas metacognitivas, regulación, conciencia metacognitiva.

\begin{abstract}
The present study is aimed at determining the influence of the IMA program on the reading comprehension of students in technical education. We used a research application type, quasi-experimental and using the Solomon design of four groups. A set of activities, related to levels of comprehension and metacognitive techniques, since in each of the 12 sessions of the program IMA applied tests of reading and metacognitive techniques. By means of a sampling not-probabilistic chose a sample of two experimental groups, each with 27 students, and two groups of control, with 24 and 31 students, respectively. The discussion of the results determined that participants in this program, students have evolved their literacy skills, based on the comparison of pre and post test results, strengthening the idea that metacognitive skills and reading comprehension makes sense, if you think on the basis of learning. Students increased significantly their metacognitive skills, since the first experimental group, from the comparative points, increased 4,45 points and in the second there was 4,15 points. Therefore, the proposal enables student to reflect on their academic commitment, the ability to understand and self-regulation that will allow you to improve your training professional technician.
\end{abstract}

Keywords: Program, reading comprehension, metacognitive skills, technical metacognitive, regulation, awareness metacognitive.

Historial del artículo:

Recibido, 15 de setiembre de 2017; aceptado,4 de enero de 2018; disponible en línea, 15 de enero de 2018

* Doctor en Ciencias, Jefe de la División de investigación de la Escuela de Oficiales de la Fuerza Aérea del Perú.

Correo: aguayomelendez@gmail.com 


\section{INTRODUCCIÓN}

En la actualidad, se reconoce que la lectura es una actividad necesaria, pero para algunos padres, es un acto sancionador y otros, una actividad para vencer el insomnio, paradójicamente. Lo preocupante que esas sensaciones se evidencie en la etapa académica de jóvenes que aspiran ser profesionales, lo cual afecta negativamente en el desarrollo cognitivo y lingüístico. En el 2016, los resultados de la evaluación Censal de Estudiantes (Ece) indicaban una mejoría en las capacidades relacionadas a la matemática, pero un retraso en los niveles de comprensión lectora ya que en el 2015, el 49,8\% de escolares de segundo de primaria entendían lo que leían, y en el 2016, se redujo a $46,4 \%$. Aunque cabe precisar y relacionar que solo tres de cada 10 jóvenes, de más de 15 años, accede a la educación superior en el Perú, equivalente al $16 \%$ de la población, según los resultados de la Estadística de la Calidad Educativa (ESCALE) del Ministerio de Educación (2015). Se dijo que uno de los factores determinantes fue la lengua materna, ya que un $30 \%$ de los que acceden a estudios superiores tienen como lengua materna el castellano y un $21 \%$ lengua indígena. Esto podría limitar comprender textos en castellano que son tentativamente, más utilizados para desarrollar las habilidades lectoras.

Por tal motivo, se debe proponer mejoras en el proceso de enseñanza-aprendizaje, partiendo de las capacidades lectoras de los estudiantes. De igual forma, se recurre a la reflexión del docente, ya que es necesario el protagonismo del estudiante y del docente mediador en los procesos de comprensión, centrando en la construcción y sistematización de conocimientos para desarrollar habilidades y estrategias que permita experimentar con instrumentos metacognitivos. Hablar de metacognición, se necesita concientizar sobre los procesos cognitivos involucrados en el aprendizaje, ayudando al estudiante en autorregular o autodirigir su proceso. En este sentido, el presente estudio buscó determinar la influencia del programa IMA en la comprensión lectora de los estudiantes, ya que en el nivel práctico aportó datos importantes para la elaboración y reestructuración de técnicas y estrategias de aprendizaje, especialmente en la reflexión de las habilidades metacognitivas en los talleres de lectura. Cabe indicar que el logro de las habilidades metacognitivas en aulas de educación superior, son necesarias para el desarrollo del pensamiento científico y una perspectiva interna sobre el proceso de aprendizaje. Además, se buscó preparar a los estudiantes en el anticipo de situaciones nuevas - enfrentar problemas que le permitan analizar deficiencias, cambios y el desarrollo de habilidades.

En los estudios realizados, se destaca la de Escalante (2008) desarrollada en la Universidad Nacional Mayor de San Marcos titulado: La comprensión lectora y el rendimiento académico entre los estudiantes de Pregrado de la Facultad de Educación de la UNMSM. Los resultados obtenidos demostraron que el nivel de comprensión lectora de los estudiantes de Pregrado de la Facultad de Educación es bajo porque se sitúa en un promedio de 12,76 en una escala vigesimal. En cambio, Ladino y Tovar (2005) desarrollaron en la Universidad Antonio Nariño de Bogotá, Colombia, titulada: Evaluación de las estrategias metacognitivas, para la comprensión de textos científicos, en la Universidad Pedagógica Nacional de Bogotá. Los resultados obtenidos demostraron que los estudiantes no precisamente poseen una estrategia totalmente estructurada para la tarea de enfrentarse a un texto científico, sino que exhiben algunos indicadores de funcionamiento metacognitivo. Sus estrategias no son efectivas en ciertos casos pues, ellas diseñaron estrategias e incluso las evaluaron para detectar sus fallas; pero en el análisis del manejo o nivel conceptual, se refleja que en varios casos estas mismas estrategias implementadas en cierta forma, no dieron resultados efectivos; pues algunas de las respuestas a los problemas planteados no fueron exitosas.

El presente estudio determinó como hipótesis: la aplicación del programa IMA mejora la comprensión lectora de los estudiantes del Instituto de Educación Superior Tecnológico Privado Franklin Roosevelt, ya que dicho programa esta basado en la aplicación de técnicas metacognitivas empleadas, durante las 12 sesiones, por los estudiantes para desarrollar habilidades metacognitivas y mejorar los niveles de comprensión de textos. Por esto, se precisa lo que dijo Flavell (1987) en relación a la lectura: "(...) como un proceso activo en el cual un lector interactúa con el texto para construir significado. En la construcción de significado es importante la conciencia del lector y el monitoreo durante el proceso de comprensión. Esta conciencia y el monitoreo se denominan metacognición". Por tanto es necesario precisar, según Tamayo y Vasco (2013) el término metacognición es "el conocimiento de los procesos cognitivos y la regulación de estos". Asimismo, Flower, citado por Bausela (2012) la metacognición es "La conciencia del individuo de sus puntos fuertes y débiles y su autoregulación".

En relación a las habilidades metacognitivas, Baker y Anderson (1981), señalados por Pinzas (2003) plantearon que cuando el individuo utiliza su saber de cómo se lee y cuáles son los procesos que mejoran la comprensión, está llevando a cabo un trabajo de monitoreo. Por tanto, la conciencia metacognitiva permite la sensación de saber y, al mismo tiempo, aplicar hábilmente el conocimiento en un contexto concreto, además de ser consciente de la utilidad de determinadas estrategias cognitivas relacionadas con el proceso lector y según el grado en el que conoce de estas y estén dispuestos aplicarlas. Incluso, Vargas y Arbeláez Gómez (2001) indicaron que las habilidades 
metacognitivas no solo se aplican a la lectura, si no también aquellas habilidades comunicativas como la escritura, escucha, habla, la resolución de problemas, el mismo estudio y otros procesos cognitivos. La metacognición genera autonomía en el aprendizaje. Más aún cuando Larraz (2015) indicó que las habilidades metacognitivas son un medio para adquirir el conocimiento metacognitivo y la regulación de las estrategias metacognitivas.

El Monitoreo y la Regulación metacognitiva implica precisar que "monitorear o guiar la comprensión mientras se está leyendo, incluye una lectura orientada a metas. Estas metas son justamente las que proporcionan un contexto significativo $\circ$ un marco de referencia que sirve de orientación al lector para seleccionar e integrar la nueva información que trae el texto" (Pinzas, 2003, p. 90). Además, la regulación incluye estrategias dirigidas ante diversos problemas de comprensión y se activa cuando es evidente para el lector que hay una falla. Estas estrategias pueden incluir releer la parte del texto que no se entiende y acciones de búsqueda, recogiendo elementos previos y posteriores que contribuyan a aclarar y solucionar el problema de comprensión. "(...) la meta cognición, el monitoreo cognitivo y el monitoreo de la comprensión son conceptos jerárquicamente relacionados. El monitoreo de la comprensión es un tipo de monitoreo cognitivo y este es un componente de la meta cognición" (Baker y Brown, 1984).

Pinzás (2003) consideró útiles, entre otras, para la evaluación continua de la comprensión lectora, como del monitoreo y regulación cognitiva: La Técnica del texto interferido que es utilizada por los que manipulan intencionalmente el grado de inteligibilidad de los textos para que sean menos coherentes y evidenciar si los lectores identifican los elementos que los confunden: el error deliberado. Además, es posible que se le pida que lo corrija y muestre como debió ser escrito. A través del texto interferido, el investigador examina si el lector está construyendo significados y si reacciona cuando estos se alteran. Cuando el texto contiene errores que contradicen las expectativas cognitivas del lector surge la disonancia cognitiva, también denominada disonancia textual. Por esta razón, Haller y sus colaboradores (1988), citada por Pinzas (2003) encontraron que "una de las mejores maneras de enseñar a los estudiantes estrategias metacognitivas es a través de la disonancia textual." La reacción de los lectores a la disonancia textual se toma como indicador de la presencia de habilidades de monitoreo cognitivo.

Otra técnica considerada útil es la Técnica Cloze donde el sujeto debe predecir una serie de palabras que han sido omitidas de manera sistemática e intencional de un texto escrito y reemplazadas por espacios en blanco. Este procedimiento obliga al lector utilizar su bagaje cognitivo, su conocimiento previo del tema, construcciones sintácticas y el contexto semántico. Desde esta perspectiva, Baker y Brown (1984), describieron varias técnicas de investigación que son empleadas para evaluar el monitoreo cognitivo en la comprensión lectora, considerando a la Técnica de tipo Cloze como una de ellas. La propiedad de su elección permite evaluar si los lectores han entendido el sentido del texto. La forma varía, ya que los lectores pueden escribir las palabras omitidas, y en otros, pueden elegirlas entre alternativas ofrecidas por el texto.

De igual manera, la evaluación textual como expresión del monitoreo cognitivo permite comparar el contenido del texto con las expectativas del lector, muchos consideran como un proceso de examen de la consistencia del texto que lo denominan evaluación textual. Cuando el lector encuentra dificultad para integrar el texto con lo que sabe o integrar partes nuevas del texto con las ya leídas, deja de comprender, pues no puede otorgar significados o generar una representación mental coherente. Cuando esto sucede, el buen lector, sin darse cuenta, realiza sus procesos de evaluación textual, para entender qué sucedió, cuál es el origen de su confusión y qué puede a hacer para empezar a solucionarla. Por tanto, se debe considerar de que el lector debe estar comprometido en su evaluación textual y considerarla como una expresión de la conciencia y el monitoreo cognitivo de la compresión.

Por último, Solé (2009) afirmó que leer es un proceso con el propósito de convertir a los lectores en autónomos, capaces de enfrentarse de manera interactiva a textos de distinta índole y capaces de aprender a partir de los textos. Además, los niveles de comprensión teniendo en cuenta que es un proceso de interacción entre el texto y el lector, Strang (1965), Jenkinson (1976) y Smith (1989) plantearon tres niveles de comprensión:

- Comprensión Literal que implica la información explícita.

- Comprensión Inferencial que implica inferencias inductivas y deductivas. "Como su nombre indica, alude a lo implícito en el texto, a las relaciones que no están explícitamente planteadas. Por ello, se relaciona con la habilidad para hacer predicciones e hipótesis de contenido para la interpretación de los personajes y sus motivaciones, para la comparación y el contraste, (...)" (Pinzas, 2001).

- Comprensión Criterial que implica emitir un juicio de valor por lo que requiere de un mayor nivel de abstracción desarrollando el pensamiento crítico ya que la validez de una opinión radica en la calidad del argumento. 


\section{MATERIAL Y MÉTODOS}

La presente investigación tuvo un enfoque cuantitativo, ya que se utilizó el análisis estadístico a partir de las pruebas e instrumentos para probar las hipótesis, siendo de tipo experimental, porque se explicó el problema existente: la deficiente comprensión lectora. Por esta razón, se evaluó la influencia del programa IMA en los niveles de comprensión lectora de los estudiantes presentándolo como una alternativa para optimizar el desempeño académico de los grupos estudiados. No se desconoce la influencia de otras variables extrañas. Además, el diseño del estudio fue cuasi experimental: "diseño con pre prueba y pos prueba a grupos intactos"; es decir, de cuatro grupos de Solomon. A los cuatro grupos se les aplicó la posprueba. Al respecto, Hernández, Fernández y Baptista (2014) dijeron: "La ventaja de este diseño es que el experimentador tiene la posibilidad de verificar los posibles efectos de la preprueba sobre la posprueba, puesto que a unos grupos se les administra un test previo y a otros no". El esquema correspondiente es:

$\begin{array}{lccc}\text { RG1 } & 01 & X & 02 \\ \text { RG2 } & 03 & - & 04 \\ \text { RG3 } & - & X & 05 \\ \text { RG4 } & - & - & 06\end{array}$

En cuanto a la población, y por la naturaleza del estudio, se consideró a los 420 estudiantes de las carreras técnicas de Farmacia y Enfermería técnica de ambos turnos: diurno y nocturno del I.E.S.T.P. Franklin Roosevelt. $Y$ en base a un muestreo de tipo intencional. Al respecto Hernández, Fernández y Baptista (2014) señalaron: "Elegir entre una muestra probabilística o una no probabilísticas depende del planteamiento del estudio, del diseño de investigación y de la contribución que se piensa hacer con ella". Por tanto, se respetó las aulas ya formadas por los estudiantes que estuvieron constituidas por 27 estudiantes cada uno, de ambos sexos, con edades promedio de 18 a 25 años, concernientes a los dos grupos del primer ciclo del turno noche. En cambio, los dos grupos del primer ciclo del turno diurno fueron los grupos de control.

Referente a las técnicas, se validó la prueba de comprensión lectora que tuvo como propósito evaluar los niveles de comprensión lectora mediante cinco Test que constó de 42 preguntas agrupado en bloques correspondientes a 17 de nivel literal, 15 de inferencial y 10 de Criterial. Cada test contiene ocho preguntas, a excepción de la primera, con diez preguntas, con cinco opciones como respuestas. Se aplicó el método de Juicio de Expertos, profesionales con grados académicos de maestro y doctor. La confiabilidad del instrumento se basó en las tres dimensiones de la comprensión lectora obteniendo el valor de 0.89, concluyendo que la prueba tiene una excelente confiabilidad. En relación al Programa IMA tuvo como propósito la evaluación de los niveles de comprensión lectora mediante 12 pruebas contando con 4 preguntas correspondientes al nivel literal, inferencial y Criterial. Así como las pruebas semiabiertas basados en la evaluación del monitoreo y regulación metacognitiva aplicando las técnicas Cloze y del Texto interferido, intercaladamente. Las pruebas de los niveles de lectura denominadas IMA 1.1 respectivamente hasta IMA 12.1. En cambio, las pruebas semiabiertas de monitoreo y regulación metacognitiva basados en las técnicas Cloze y del Texto interferido son denominadas IMA 1.2 respectivamente hasta IMA 12.2.

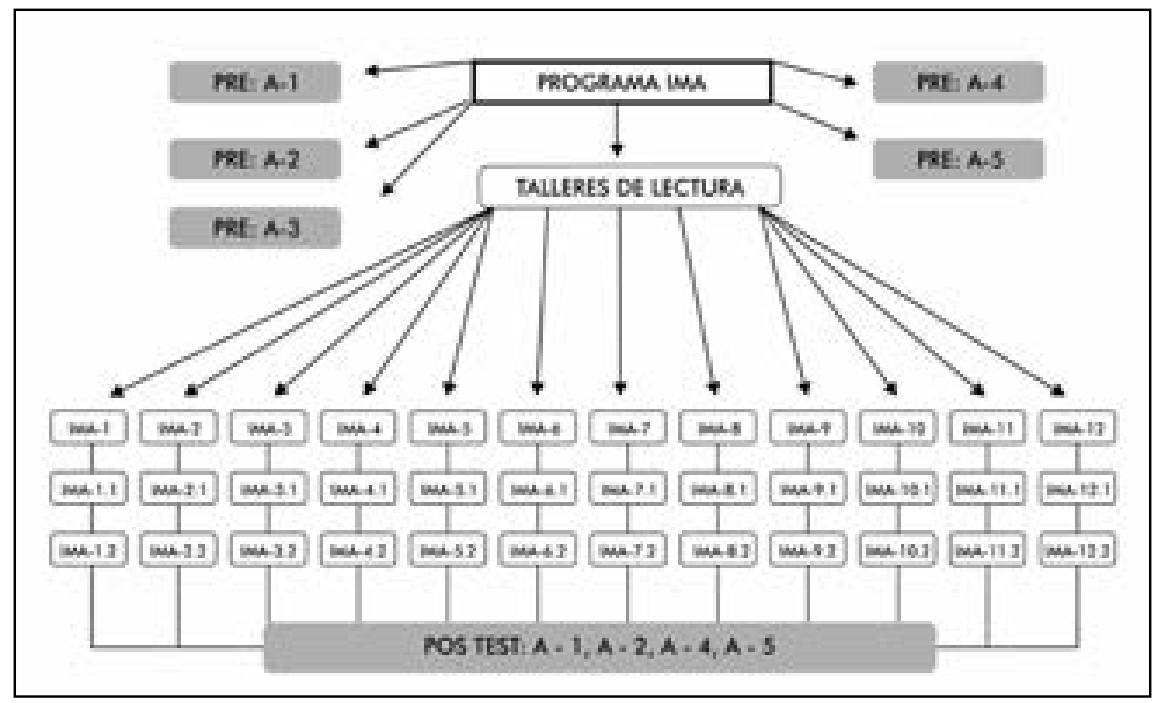

Figura 1. Esquema de instrumentos del Programa IMA. 


\section{RESULTADOS}

En el presente apartado se muestra el análisis y discusión de los resultados de la investigación, los cuales fueron originados de la recolección de información por medio de la aplicación de las pre y pos test así como las 12 pruebas de evaluación de los niveles de comprensión lectora y 12 pruebas semiabiertas basados en la evaluación del monitoreo y regulación metacognitiva aplicando las técnicas Cloze y del Texto interferido, intercaladamente. En la Tabla 1, se detalla aspectos relevantes.

Teniendo en cuenta que el puntaje máximo teórico de la prueba Comprensión lectora es de 20 puntos, se observó que el grupo experimental con pre test (10.58 como media) tiene un nivel de comprensión lectora ligeramente menor que el grupo control (12.52). Asimismo, ambos grupos tienen la misma cantidad de dispersión de las puntuaciones de sus integrantes alrededor de sus medias aritméticas (D. S. de 1.31 y 1.26). Descriptivamente en el post test, se observó que tanto el grupo experimental con pre test como el grupo experimental sin pre test, tienen una puntuación media bastante cercana al puntaje máximo teórico de alcanzar en la prueba de comprensión lectora (20); es decir, que ambos tienen similares medias aritméticas en la referida comprensión (18.56 y 18.25, respectivamente). Además ambas medias son más elevadas que de los grupos control, sea con pre test o sin pre test (13.82 y 12.55 , respectivamente). Puede decirse que los grupos experimentales en el pos test lograron niveles altos de comprensión lectora, y los grupos control lograron un nivel medio (Tabla 2).

Los grupos experimentales con pre test y sin pre test, difieren en un nivel estadístico muy significativo $(p<$ 0.000 ) de los grupos control con pre test y sin pre test. En consecuencia, se rechazó la hipótesis nula ya que el Programa IMA ha demostrado ser efectivo en el

Tabla 1

Comparación de medias del pre-test y pos-test en todos los grupos.

\begin{tabular}{lccccc}
\hline \multicolumn{1}{c}{ Grupos } & $\mathrm{N}$ & Media pre-test & D.S. & Media pos-test & D.S. \\
\hline $\begin{array}{l}\text { Grupo Experimental } \\
\text { con pre-test }\end{array}$ & 27 & 10,58 & 1.31 & 118,56 & 0,76 \\
$\begin{array}{l}\text { Grupo control con } \\
\text { pre-test }\end{array}$ & 30 & 12,52 & 1.26 & 13,82 & 1,23 \\
$\begin{array}{l}\text { Grupo experimental } \\
\text { sin pre-test }\end{array}$ & 27 & no aplicado & - & 18,25 & 0,60 \\
$\begin{array}{l}\text { Grupo control sin } \\
\text { pre-test }\end{array}$ & 24 & no aplicado & - & 12,55 & 0,85 \\
Total & 108 & 11,55 & & 15,83 & \\
\hline
\end{tabular}

Tabla 2

Prueba Post-hoc de los resultados del pos-test en todos los grupos.

\begin{tabular}{|c|c|c|c|}
\hline \multicolumn{4}{|c|}{ Resultados del pos-test - Bonferroni } \\
\hline (I) Grupos evaluados & (J) Grupos evaluados & Diferencia de medias (I-J) & Sig. \\
\hline \multirow{3}{*}{$\begin{array}{l}\text { Grupo Experimental } \\
\text { con pre-test }\end{array}$} & Grupo control con pre-test & 4.74 & 0,00 \\
\hline & $\begin{array}{l}\text { Grupo experimental sin } \\
\text { pre-test }\end{array}$ & 0.31 & 1,00 \\
\hline & Grupo control sin pre-test & 6.01 & 0,00 \\
\hline \multirow[t]{3}{*}{$\begin{array}{l}\text { Grupo control con } \\
\text { pre-test }\end{array}$} & $\begin{array}{l}\text { Grupo Experimental con } \\
\text { pre-test }\end{array}$ & -4.74 & 0,00 \\
\hline & $\begin{array}{l}\text { Grupo experimental sin } \\
\text { pre-test }\end{array}$ & -4.43 & 0,00 \\
\hline & Grupo control sin pre-test & 1.27 & 0,00 \\
\hline \multirow[t]{2}{*}{$\begin{array}{l}\text { Grupo experimental } \\
\text { sin pre-test }\end{array}$} & $\begin{array}{l}\text { Grupo Experimental con } \\
\text { pre-test }\end{array}$ & -0.31 & 1,00 \\
\hline & $\begin{array}{l}\text { Grupo control con pre-test } \\
\text { Grupo control sin pre-test }\end{array}$ & $\begin{array}{l}4.43 \\
5.70\end{array}$ & $\begin{array}{l}0,00 \\
0,00\end{array}$ \\
\hline \multirow[t]{2}{*}{$\begin{array}{l}\text { Grupo control sin } \\
\text { pre-test }\end{array}$} & $\begin{array}{l}\text { Grupo Experimental con } \\
\text { pre-test }\end{array}$ & -6.01 & 0,00 \\
\hline & $\begin{array}{l}\text { Grupo control con pre-test } \\
\text { Grupo experimental sin } \\
\text { pre-test }\end{array}$ & $\begin{array}{l}-1.27 \\
-5.70\end{array}$ & $\begin{array}{l}0,00 \\
0,00\end{array}$ \\
\hline
\end{tabular}


incremento de la comprensión lectora en términos generales.

En base a los dos grupos experimentales donde se implementó el programa IMA, los resultados de las pruebas del grupo 1, relacionados a las habilidades metacognitivas en cada sesión se muestran en la tabla 3.

En la tabla se observa la progresión de los promedios de las habilidades metacognitivas durante las 12 sesiones. Se muestra en la última, un incremento de
En la tabla 4 se observa la progresión de los promedios de los niveles de comprensión lectora en las 12 sesiones. Se muestra que en esta última hay un incremento de 4,58 puntos respecto a la sesión 1, precisando las medias de 13,52 en la primera y 18,1, en la última. Por tal motivo, se consideró conveniente comparar las sesiones 1 y 6 y 1 y 12 . Al tratarse de mismo grupo se utilizó la prueba $\mathrm{T}$ de Student para muestras correlacionadas comparando los promedios de los niveles de comprensión lectora se alcanzó un resultado estadísticamente significativo (p $<0,000)$. Por ende, se rechaza la hipótesis nula y

Tabla 3

Progresión de las habilidades metacognitivas en el Grupo 1.

\begin{tabular}{lcccccccccccc}
\hline \multicolumn{11}{c}{ Sesiones } \\
\hline Estadísticos & 1 & 2 & 3 & 4 & 5 & 6 & 7 & 8 & 9 & 10 & 11 & 12 \\
\hline Media & 10,22 & 11,11 & 8,89 & 11,81 & 12,67 & 14,70 & 15,74 & 15,78 & 10,81 & 13,04 & 13,78 & 14,67 \\
$\begin{array}{l}\text { Desviación } \\
\text { Estándar }\end{array}$ & 4,61 & 3,73 & 3,08 & 2,03 & 2,86 & 3,12 & 1,91 & 1,82 & 4,68 & 2,37 & 3,20 & 2,71 \\
$\mathrm{~N}$ & 27 & 27 & 27 & 27 & 27 & 27 & 27 & 27 & 27 & 27 & 27 & 27 \\
\hline
\end{tabular}

Tabla 4

Progresión de los niveles de comprensión lectora en el Grupo 1.

\begin{tabular}{lcccccccccccc}
\hline \multicolumn{11}{c}{ Sesiones } \\
\hline Estadísticos & 1 & 2 & 3 & 4 & 5 & 6 & 7 & 8 & 9 & 10 & 11 & 12 \\
\hline Media & 13,52 & 12,59 & 14,63 & 14,44 & 16,5 & 16,1 & 18,43 & 18,52 & 18,7 & 17,4 & 18,8 & 18,1 \\
$\begin{array}{l}\text { Desviación } \\
\text { Estándar }\end{array}$ & 3,62 & 4,54 & 4,58 & 4,23 & 2,82 & 2,82 & 2,71 & 2,70 & 2,83 & 3,5 & 2,11 & 2,82 \\
$\mathrm{~N}$ & 27 & 27 & 27 & 27 & 27 & 27 & 27 & 27 & 27 & 27 & 27 & 27 \\
\hline
\end{tabular}

Tabla 5

Progresión de las habilidades metacognitivas en el Grupo 2.

\begin{tabular}{lcccccccccccc}
\hline \multicolumn{10}{c}{ Sesiones } \\
\hline Estadísticos & 1 & 2 & 3 & 4 & 5 & 6 & 7 & 8 & 9 & 10 & 11 & 12 \\
\hline Media & 10,81 & 12,30 & 12,48 & 11,74 & 15,48 & 13,11 & 11,96 & 12,11 & 9,04 & 12,00 & 10,81 & 14,96 \\
$\begin{array}{l}\text { Desviación } \\
\text { Estándar }\end{array}$ & 3,24 & 3,12 & 2,35 & 1,95 & 2,65 & 3,40 & 2,51 & 3,87 & 3,25 & 2,93 & 3,29 & 3,05 \\
$\mathrm{~N}$ & 27 & 27 & 27 & 27 & 27 & 27 & 27 & 27 & 27 & 27 & 27 & 27 \\
\hline
\end{tabular}

4,45 puntos con respecto a la sesión 1 , precisando las medias de 10,22 en la primera y 14,67, en la última. Por tanto, se consideró conveniente comparar las sesiones 1 y 6 y 1 y 12 . Al tratarse de mismo grupo, se utilizó la prueba T de Student para muestras correlacionadas comparando los promedios de las habilidades metacognitivas de las sesiones 1 y 6 y de las sesiones 1 y 12 , lo que alcanzó un resultado estadísticamente muy significativo $(p<0,000)$. Por tanto, se rechaza en ambos casos la hipótesis nula y se acepta la Hipótesis Alterna. El programa IMA ha tenido un efecto positivo incrementando las habilidades metacognitivas. se acepta la Hipótesis Alterna. El programa IMA ha tenido un efecto positivo incrementando los niveles de comprensión lectora.

En base a los dos grupos experimentales donde se implementó el programa IMA, los resultados de las pruebas del grupo 2, relacionados a las habilidades metacognitivas en cada sesión se muestran en la tabla 5. En esta tabla se observa la progresión de los promedios de las habilidades metacognitivas en las 12 sesiones que duró el programa IMA. Se encuentra que en esta última hay un incremento de 4,15 puntos 
Tabla 6

Progresión de los niveles de comprensión lectora en el Grupo 2.

\begin{tabular}{lcccccccccccc}
\hline \multicolumn{10}{c}{ Sesiones } \\
\hline Estadísticos & 1 & 2 & 3 & 4 & 5 & 6 & 7 & 8 & 9 & 10 & 11 & 12 \\
\hline Media & 10,74 & 10,56 & 14,63 & 14,81 & 14,26 & 15,7 & 19,26 & 18,33 & 18,89 & 19 & 19 & 19,6 \\
$\begin{array}{l}\text { Desviación } \\
\text { Estándar }\end{array}$ & 5,13 & 4,0 & 2,37 & 2,18 & 4,31 & 3,12 & 1,81 & 1,82 & 2,11 & 1,97 & 1,97 & 1,33 \\
$\mathrm{~N}$ & 27 & 27 & 27 & 27 & 27 & 27 & 27 & 27 & 27 & 27 & 27 & 27 \\
\hline
\end{tabular}

respecto a la sesión 1, precisando las medias de 10,81 en la primera y 14,96 , en la última. Se consideró conveniente comparar las sesiones 1 y 6 y 1 y 12 . Al tratarse de mismo grupo se utilizó la prueba $T$ de Student para muestras correlacionadas comparando los promedios de las habilidades metacognitivas, existen diferencias estadísticas significativas $(p<$ $0,684)$ entre 1 y $6 ;(p<0,009)$ entre 1 y 12 a favor de 12. Por tanto, se rechaza en ambos casos la hipótesis nula y se acepta la Hipótesis Alterna. El programa IMA ha tenido un efecto positivo incrementando las habilidades metacognitivas.

En la tabla 6 se observa la progresión de los promedios de los niveles de comprensión lectora en las 12 sesiones que duró el programa IMA. Se encuentra que en esta última hay un incremento de 8,86 puntos respecto a la sesión 1, precisando las medias de 10,74 en la primera y 19,6 , en la última. Por tanto, se consideró conveniente comparar las sesiones 1 y 6 y 1 y 12 . Al tratarse de mismo grupo se utilizó la prueba $T$ de Student para muestras correlacionadas comparando los promedios de los niveles de comprensión lectora se alcanzó un resultado estadísticamente significativo ( $p$ $<0,000)$. Por tal motivo, se rechaza la hipótesis nula y se acepta la Hipótesis Alterna. El programa IMA ha tenido un efecto positivo incrementando los niveles de comprensión lectora.

\section{DISCUSIÓN}

Existen autores que respaldan de alguna manera los resultados, como es el caso de Pardo (2015) que desarrolló su investigación en la Universidad Pedagógica y tecnológica de Colombia, titulada: "Estrategias autorreguladoras para la comprensión de textos académicos en los estudiantes universitarios de la faculta de estudios a Distancia de la Universidad Pedagógica y tecnológica de Colombia". Los resultados indican que las estrategias autorreguladoras juegan un papel clave en el éxito para la comprensión no solo de textos académicos sino de diferentes tipos, ya que la autorregulación posibilita que el lector desarrolle habilidades para saber si logró el propósito de la lectura que hizo y como lo está logrando.

Los resultados del estudio comprueba que existe una influencia del Programa IMA con los niveles de comprensión lectora de los estudiantes a través de la aplicación de pruebas basado en los niveles de comprensión lectora y estrategias metacognitivas, buscando fomentar habilidades metacognitivas como la conciencia, el monitoreo y regulación metacognitiva. Entre estas estrategias se utilizó pruebas basadas en la disonancia textual. Haller y sus colaboradores (1988), citados por Pinzas (2003), encontraron que "una de las mejores maneras de enseñar a los estudiantes estrategias metacognitivas es a través de la disonancia textual". Dicho programa permitió observar la evolución académica de los participantes relacionado a sus habilidades lectoras, más aun cuando se hizo la comparación de los resultados del pre y pos test, ya que los resultados estadísticos basado en el nivel inferencial, permitió realizar las mediciones y comparaciones necesarias teniendo como una conclusión del estudio, que el Programa IMA influye significativamente en la comprensión lectora de los estudiantes, basados en las diferencias de los resultados del post test, tantos en los grupos experimental con pre test y sin pre test (tabla 2).

Llevar a cabo el desarrollo de las habilidades metacognitivas constituye un reto, por su influencia en la comprensión lectora de los estudiantes, por la confrontación entre distintos enfoques y procesos de implementación. Cabe mencionar que Baker y Brown (1984) describen técnicas que son empleadas para evaluar el monitoreo cognitivo en la comprensión de la lectura, considerando a la Técnica de tipo Cloze como una manera de evaluar si los lectores han entendido el sentido del texto.

Contribuir en este debate, se busca abrir posibilidades, ampliar miradas y demostrar que hay más formas de fortalecer la idea de que las habilidades metacognitivas y la comprensión lectora tienen sentido si se piensan en función del aprendizaje independiente de los estudiantes. "Las habilidades metacognitivas son aplicables a la lectura, a la escritura, el habla, la escucha, el estudio, la resolución de problemas y cualquier otro dominio en el que intervengan procesos cognitivos" (Vargas y Arbeláez, 2009).

Se evidenció la necesidad que tienen los estudiantes para desarrollar las habilidades metacognitivas y mejorar su comprensión lectora. Flavell (1987) en 
relación a la lectura señaló: "(...) como un proceso activo en el cual un lector interactúa con el texto para construir significado. En la construcción de significado es importante la conciencia del lector y el monitoreo durante el proceso de comprensión. Esta conciencia y el monitoreo se denominan metacognición". A través de los dos grupos experimentales del estudio, se apreció la evolución de las habilidades metacognitivas mediante las pruebas de lecturas relacionados a las técnicas metacognitivas, representado en las medias de la primera sesión comparando con la decimosegunda sesión, respectivamente. Más aún cuando la diferencia entre la media obtenida en el pos test por el grupo experimental y la obtenida por el de control es altamente significativa. Por tanto, una de las conclusiones del estudio fue que el Programa IMA incrementa de manera significativa las habilidades metacognitivas en los estudiantes; es decir en el primer grupo experimental, a partir de los puntos comparativos, hay un incremento de 4,45 puntos (Tabla 3) y en el segundo grupo experimental, 4,15 puntos (tabla 5). Se puede decir, por consiguiente, que las estrategias metacognitivas a través de la pruebas del texto interferido y del tipo Cloze mejoran los niveles de comprensión lectora de los estudiantes.

También se evidenció la necesidad que tienen los estudiantes de realizar una autoevaluación para mejorar sus niveles de comprensión lectora, a través de su participación y la actitud de controlar sus calificaciones en cada taller. "Un estudiante que es capaz de auto regular su proceso, podrá comprender y resolver de manera eficiente la tarea o problema propuesto" (Cadavid, 2014). Además, Baker y Anderson (1981), citados por Pinzás (2003), han planteado que cuando el individuo utiliza su saber de cómo se lee y sobre cuáles son los procesos que mejoran la comprensión está llevando a cabo un trabajo de monitoreo. La conciencia metacognitiva da la sensación de saber y al mismo tiempo poder aplicar hábilmente ese conocimiento en un contexto de realidad concreta, además es consciente de la utilidad de determinadas estrategias cognitivas relacionadas con el proceso lector. Además, se debe tomar conciencia que todo proceso académico necesita la madurez y la reflexión de las consecuencias de sus actos. De esta manera, se desarrolló los niveles de comprensión lectora necesarios en los estudiantes que conformaron la muestra, evidenciados en los resultados del post test, así como la influencia del programa IMA.

\section{REFERENCIAS BIBLIOGRÁFICAS}

Baker, L. y Brown, A. L. (1984). Las habilidades metacognitivas en la lectura. En P. D. Pearson, M. Kamil, R. Barr y P. Mosenthal (eds), Manual de Investigación de la lectura, pp. 353394. Asociación Internacional de Lectura.

Bausela, E. (2012) Metacognición en relación a la escritura. Madrid, España: Dykinson

Cadavid, V. (2014) Relaciones entre la metacognición y el pensamiento viso espacial en el aprendizaje de la estereoquímica. Colombia: Universidad Autónoma de Manizales

Escalante, M. (2008). La comprensión lectora y el rendimiento académico entre los estudiantes de Pregrado de la Facultad de Educación de la UNMSM. Lima, Perú: UNMSM.

Flavell, J. (1987) La especulación sobre la naturaleza y el desarrollo de la metacognición. En F.E. Weinert y R. H. Kluwe (Eds), Metacognición, motivación y comprensión (pp. 21-29). Hillsdale, N.J., Erlbaum.

Hernández, R., Fernández, C. y Baptista, P. (2014). Metodología de la Investigación. México: McGrawHill Interamericana.

Jenkinson, M. D. (1976) Modos de enseñar, en Staiger, R. C. (comp.), La enseñanza de la lectura, Buenos Aires, Argentina: Huemul

Ladino, O. y Tovar, J. C. (2005). Evaluación de las estrategias metacognitivas, para la comprensión de textos científicos. Bogotá: Universidad Pedagógica Nacional. Recuperado de http://www.unne.edu. ar/institucional/documentos/lecturayescritura08/ ladino_tovar.pdf

Larraz, N. (2015) Desarrollo de las habilidades creativas y metacognitivas en la educación secundaria obligatoria. Madrid, España: Dykinson

Minedu (2016) Resultados de la evaluación Censal de Estudiantes (Ece) Lima, Perú: Oficina de Medición de la Calidad de los Aprendizajes.

Minedu (2015) Resultados de la Estadística de la Calidad Educativa (ESCALE) Lima, Perú

Pardo, N. E. (2015) Estrategias autorreguladoras para la comprensión de textos académicos en los estudiantes universitarios. Revista Horizontes Pedagógicos, 17(2), 29-38.

Pinzas G., J. (2001). Leer pensando. Introducción a la visión contemporánea de la lectura. Lima, Perú: Pontificia Universidad Católica del Perú.

Pinzas, J. (2003). Metacognición y lectura. Lima: Fondo editorial de la pontificia Universidad Católica del Perú.

Smith, C. B. (1989) La enseñanza de la lectoescritura: un enfoque interactivo. Madrid, España: Aprendizaje Visor

Solé, I. (2009). Motivación y lectura. En Sinergias en torno a la lectura. En Aula de Innovación Educativa, $179,56-59$.

Strang, R. (1965), Procesos del aprendizaje infantil. Buenos Aires, Argentina: Paidós

Tamayo, O. E. y Vasco, C. E. (2013) Diseño y Análisis de Unidades Didácticas desde una Perspectiva Multimodal. En La clase Multimodal y la Formación y Evolución de Conceptos Científicos a través del uso de Tecnologías de la Información y 
la Comunicación (pp. 103-134). Universidad Autónoma de Manizales

Vargas, E. y Arbeláez Gómez, M. C. (2001) Consideraciones teóricas acerca de la metacognición. Revista de Ciencias Humanas, 28. Recuperado de http://www.utp.edu. $\mathrm{co} /$ chumanas/revistas/revistas/rev28/vargaS
Vargas, E. y Arbeláez Gómez, M. C. (2009) Consideraciones teóricas acerca de la metacognición, Revista Educación y cultura. 5:(2), pp. 18-36 